\title{
On Minimal Perimeter Polyminoes ${ }^{\star}$
}

\author{
Yaniv Altshuler ${ }^{1}$, Vladimir Yanovsky ${ }^{1}$, Daniel Vainsencher ${ }^{1}$, \\ Israel A. Wagner ${ }^{1,2}$, and Alfred M. Bruckstein ${ }^{1}$ \\ ${ }^{1}$ Computer Science Department, Technion, Haifa 32000 Israel \\ \{yanival@cs, volodyan@cs, danielv@tx, wagner@cs, \\ freddy@cs . technion.ac.il \\ 2 IBM Haifa Labs, MATAM, Haifa 31905 Israel \\ wagner@il.ibm.com
}

\begin{abstract}
This paper explores proofs of the isoperimetric inequality for 4-connected shapes on the integer grid $\mathbb{Z}^{2}$, and its geometric meaning. Pictorially, we discuss ways to place a maximal number unit square tiles on a chess board so that the shape they form has a minimal number of unit square neighbors. Previous works have shown that "digital spheres" have a minimum of neighbors for their area. We here characterize all shapes that are optimal and show that they are all close to being digital spheres. In addition, we show a similar result when the 8-connectivity metric is assumed (i.e. connectivity through vertices or edges, instead of edge connectivity as in 4-connectivity).
\end{abstract}

\section{Introduction}

The isoperimetric inequality for $\mathbb{R}^{2}$ states that the area enclosed by a closed simple curve is at most that enclosed by a circle of the same length, with equality occurring only for curves that are circles. This implies two conclusions about circles that are equivalent in the continuous case, but distinct in discrete spaces. It is clear that among closed simple curves of a certain length, a circle encloses a maximal area, and on the other hand, that among curves enclosing a certain area, a circle has minimal length. For discrete spaces there are special shapes that have been proved to have minimal "perimeter", for various definitions of the perimeter, corresponding to the first conclusion. In the context of the $\mathbb{Z}^{n}$ grid, Wang and Wang [1] presented an ordering of grid points, such that every finite prefix of the sequence forms a set with minimal boundary size for that cardinality. Similar arguments have been applied to $B^{n}$ (the hypercube of dimension $n$ ) and other classes of spaces, and are reviewed by Bezrukov [2]. More results appear in [3, 4, 5].

This paper is concerned with shapes that are optimal in both having minimal boundaries and having maximal areas given their boundary size. In this way, they are similar to disks. We limit our treatment to the 2 dimensional grid, and provide a characterization of shapes that are optimal in this "double" sense.

\footnotetext{
* This research was supported in part by the Israeli Ministry of Science Infrastructural Grant No. 3-942 and in part by the Devorah fund.
} 
We will start with a useful result that illustrates the differences between the double optimality we require and the weak optimality that was imposed before. We seek a tight lower bound on the size of the neighborhood of a general subset of the $\mathbb{Z}^{2}$ grid for which we know the cardinality i.e. the area. This is clearly a form of isoperimetric inequality. Bounds of this sort were used to prove lower bounds on the efficiency of a multi-agent algorithm for sweeping dynamically growing shapes [6].

Let $A$ be a finite subset of the $\mathbb{Z}^{2}$ grid. We define its neighborhood as $N(A)=$ $\left\{p \in \mathbb{Z}^{2} \mid d(p, A)=1\right\}$, where $d$ is the Manhattan metric $d((a, b),(x, y))=|a-x|$ $+|b-y|$. Then the lower bound we seek can be written in the form of an integer sequence $n(k): \mathbb{N} \rightarrow \mathbb{N}$, defined via $n(k)=\min _{|A| \geq k}\{|N(A)|\}$.

Let us first look at the 2 dimensional case of the sequence described by Wang and Wang in [1. Every prefix of this sequence is a set of tiles (a shape) that can be described as the union of a discrete sphere (all tiles whose coordinates sum to at most $k$ ) and part of the shell needed for the next largest sphere (some of the tiles whose coordinates sum to $k+1)$. The first elements of the Wang ${ }^{2}$ sequence are $(0,0),(0,1),(1,0),(-1,0),(0,-1),(1,1),(-1,1)$. The corresponding shapes can be seen in Figure 11. Because $\mathrm{Wang}^{2}$ show that the shape formed by every such prefix has a minimal boundary size for its area, a formula to calculate the neighborhood size of every such shape would provide us with a way to calculate $n(k)$. Geometrically we can say that the boundary size changes whenever the expansion of the outer shell enters a new quadrant.

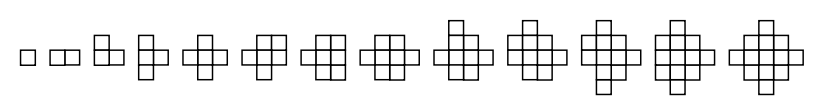

Fig. 1. The first few shapes in the 2D Wang sequence

In our approach, we first note that the function $n(k)$ is not affected by a shape that has a non minimal neighborhood size for its area (because it will not be chosen in the min), nor by a shape that has non maximal neighborhood area (since then the shape of maximal area can be used instead). Then at the beginning of the next section we provide an explicit expression for $n(k)$, which we later justify by characterizing the set of shapes that are simultaneously optimal in both having largest area for the given neighborhood size and having smallest neighborhood size given their area.

The rest of the paper is organized as follows - section 2 contains a detailed analysis of the above while section 3 presents an alternative method of producing similar results. This alternative approach is later used with slight modifications to derive similar results under the 8-connectivity metric.

\section{The Isoperimetric Inequality Theorem}

We shall next provide the promised explicit expression for $n(k)$, whose first few values are $0,4,6,7,8,8,9$. This sequence already highlights the fact that the 
fourth shape in the Wang ${ }^{2}$ sequence (Figure 1) obviously does not have maximal area for its boundary size, because $n(4)=n(5)=8$.

Theorem 1. If $k=0$, then $n(k)=0$. For $k>0 n(k)=4(m+1)+i$ where $(m, i) \in \mathbb{N} \times \mathbb{Z}_{4}$ is the first pair for which one of the following holds:

1. $i=0 \wedge k \leq 2 m^{2}+2 m+1$

2. $i=1 \wedge k \leq 2 m^{2}+3 m+1$

3. $i=2 \wedge k \leq 2 m^{2}+4 m+2$

4. $i=3 \wedge k \leq 2 m^{2}+5 m+3$

where $\mathbb{N} \times \mathbb{Z}_{4}$ is ordered lexicographically (with priority to $\mathbb{N}$ ).

While the formula for $n(k)$ in the above theorem is explicit, one might find this expression somewhat difficult to grasp. However it is easy to understand as a way to fill a simple look up table, the first few columns of which are shown below and which is scanned column first for the first value above or equal to $k$. Then the column and row of that value provide $i$ and $m$ needed to calculate the perimeter.

\begin{tabular}{|c|c|c|c|c|c|c|c|c|c|}
\hline$i \backslash m$ & 0 & 1 & 2 & 3 & 4 & 5 & 6 & 7 & 8 \\
\hline 0 & 1 & 5 & 13 & 25 & 41 & 61 & 85 & 113 & 145 \\
\hline 1 & 1 & 6 & 15 & 28 & 45 & 66 & 91 & 120 & 153 \\
\hline 2 & 2 & 8 & 18 & 32 & 50 & 72 & 98 & 128 & 162 \\
\hline 3 & 3 & 10 & 21 & 36 & 55 & 78 & 105 & 136 & 171 \\
\hline
\end{tabular}

Note that the increasing sequence of values that appear in the successive rows of the table, i.e. $123568101315 \ldots$ etc. are the areas of the double optimal shapes.

To gain some geometrical understanding of $n(k)$ in terms of $m$ and $i$, we reconsider the Wang ${ }^{2}$ sequence. This sequence includes among others, some optimal shapes, which are those whose area appears in the table above. As we mentioned, such a shape can be seen as a digital sphere enclosed by a shell of zero to three quadrants. It is easy to see by continuing the sequence that the radius of the digital sphere is $m+1$ and the number of quadrants is $i$. A complete correspondence of these areas to all optimal shapes, including small ones, will be proved in the next sections.

Our exploration of optimal shapes that yields theorem 1 consists of two phases. First we show that the optimal shapes belong to a class of simple shapes (section 2.1) and explore the structure common to all the shapes of this class (sections 2.2 and 2.3). Then we use this structure to find which shapes in that class are indeed optimal (section 2.4 ).

\subsection{Simple Shapes}

In this section we present an algorithm that allows us to cover every shape with a simple shape of the same neighborhood size and at least as much area. This will show that only simple shapes may be optimal. We will show how to calculate the neighborhood size of a simple shape, and later on its area. 
Definition 1. $(x, y)$ is called a 4 neighbor of $A$ if :

$$
(x, y) \notin A \text { and }\{(x, y+1),(x+1, y),(x-1, y),(x, y-1)\} \cap A \neq \emptyset
$$

The set of 4 neighbors of $A$ is written $N(A)$.

Definition 2. A shape $A$ is called optimal if for every shape $B$ :

$$
(|N(B)| \leq|N(A)| \Rightarrow|B| \leq|A|) \text { and }(|B| \geq|A| \Rightarrow|N(B)| \geq|N(A)|)
$$

Definition 3. A shape is called simple if it can be written as :

$$
B=\left\{(x, y) \mid y-x \in\left[j_{1}, j_{2}\right] \text { and } x+y \in\left[k_{1}, k_{2}\right]\right\}
$$

We often refer to the sizes of a simple shape as $j=j_{2}-j_{1} ; k=k_{2}-k_{1}$. Sometimes the specific directions do not matter, in which cases we denote w.l.o.g $a=\min \{j, k\} ; b=\max \{j, k\}$. Note that there may be different shapes that have the same dimensions.

Theorem 2. If $A$ is optimal, then $A$ is simple.

Proof. Let $A=\{(x, y)\}$ be a set of tiles, $k_{1}=\min \{k \mid \exists(x, y) \in B \wedge x-y=k\}$, $k_{2}=\max \{k \mid \exists(x, y) \in B \wedge x-y=k\}, j_{1}=\min \{j \mid \exists(x, y) \in B \wedge x+y=j\}$, and $j_{2}=\max \{j \mid \exists(x, y) \in B \wedge x+y=j\}$.
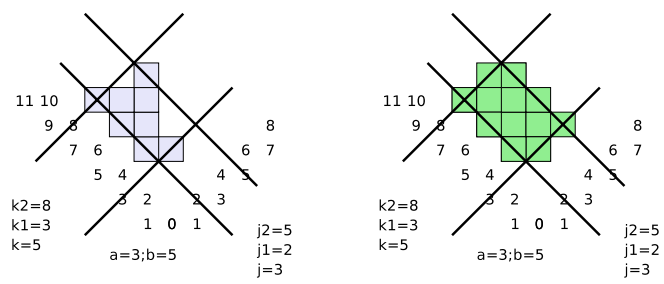

Fig. 2. A general shape $A$ and the corresponding simple shape $B$

We look at the shape $B=\left\{(x, y) \mid x-y \in\left[j_{1}, j_{2}\right] \wedge x+y \in\left[k_{1}, k_{2}\right]\right\}$, then clearly $B \supset A$. We will show that if $A$ is optimal, $B=A$. Since $B$ is simple, this is sufficient. On each boundary line there is at least one point that is in $A$. Let $\left\{\left(x_{1}, y_{1}\right),\left(x_{2}, y_{2}\right),\left(x_{3}, y_{3}\right),\left(x_{4}, y_{4}\right)\right\}$ be on such a boundary, where $\left(x_{i}, y_{i}\right),\left(x_{i+1}, y_{i+1}\right)$ are on non-opposite sides. Note that it is possible that $\left(x_{i}, y_{i}\right)=\left(x_{i+1}, y_{i+1}\right)$, for example for $A^{\prime}=\left\{\left(x_{0}, y_{0}\right)\right\}$ all the points are the same, and that we consider the indexes $i$ modulo 4 , so that $i=4 \Rightarrow i+1=1$.

W.l.o.g, we assume that $y_{i}-x_{i}=k_{2} \wedge x_{i+1}+y_{i+1}=j_{2}$, then $x_{i} \leq x_{i+1}$. Since there are no vacant columns between $x_{i}, x_{i+1}, A$ has at least $x_{i+1}-x_{i}+1$ neighbors from above (in each column, the neighbor above the highest tile of $A$ in that column - see Figure 3). Doing the same for the other 3 adjacent pairs of points, we find a lower bound on neighbors from the left, from below, and from the right. Note that this bound is tight for shape $B$, which has no other neighbors, and has all the possible tiles. Then if $A \neq B, A$ is not optimal, because it has at least as many neighbors, and not as many tiles. 
Definition 4. A simple shape $A$ with $a=0$ is denoted as degenerate shape.

Note that degenerate shapes behave differently from other simple shapes (for example, in the degenerate case $b$ cannot have odd values, because the Manhattan distance between two tiles on a diagonal is always even).

Lemma 1. The only optimal degenerate shapes have an area of 0, 1 or 2.

Proof. If $A$ is degenerate, then $a=0$. We assume by contradiction that $b \geq 4$ (see Figure 3) and $A$ is optimal. But the shape $B$, created by placing all the tiles in the same column has exactly as many neighbors (two horizontal neighbors per tile, and two additional vertical neighbors), and the same area, but is not simple, therefore is not optimal. Then $A$ cannot be optimal. The shapes with $b \leq 3$ have areas as described, as can be seen in Figure 7.

Since we have seen that only a small and finite set of degenerate shapes is of interest to our discussion we shall assume that all simple shapes have $a \geq 1$.

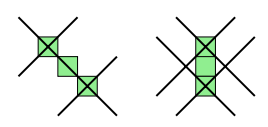

Fig. 3. A degenerate shape $A$, and a variation $A^{\prime}$ which clarifies that $A$ is non optimal

Lemma 2. Every simple shape has $j+k+4$ 4-Neighbors

Proof. By induction on $j$ and $k$. This is true for the shape of two neighboring tiles (i.e $j=k=1$ ), and 6 neighbors. In the induction step, we assume validity for $j, k$ and prove it for $j+1$ (same reasoning applies to expansion in $k$ ). Adding 1 to $j$ causes one diagonal side (having $r$ tiles) to expand to some direction (an expansion up is illustrated in Figure 4). As a result, $r$ neighbors in that direction become new tiles, and $r$ vacant beyond those in the same direction become neighbors, not modifying the neighborhood size yet. However, the new tile that is last in the direction of advancement is exposed to a new neighbor from the side. Having been diagonal to an extreme tile in the shape, it was not a neighbor before (i.e. increasing $j$ or $k$ adds one neighbor), thus a shape of dimensions $(j+1), k$ has $j+1+k+4$ neighbors, completing the induction step.

\subsection{Expansion}

In this section we demonstrate how each simple shape can be described as a "spine" expanded by an iterative expansion process. This process and its effects on the area and neighborhood size of a simple shape is described.

Definition 5. Let $A$ be a simple shape of dimensions $j, k$. We call increasing each of $j, k$ by two an expansion step. 

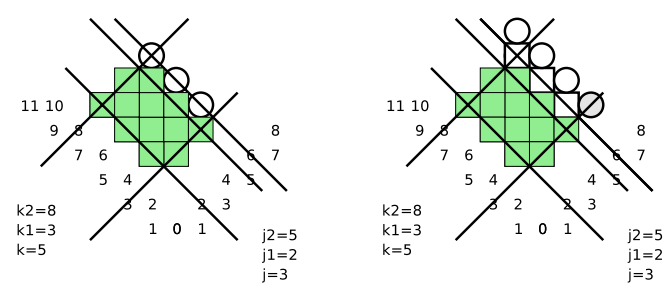

Fig. 4. Expanding a simple shape to one side

Note that each expansion step performed on a simple shape adds exactly all of its 4-neighbors. Thus, the number of tiles of the shape increases by $j+k+4$, and the number of neighbors grows by 4 . See Figure 5 for an example.

Lemma 3. Let $A$ be a simple shape with dimensions $j, k$. After $s$ expansion steps, its neighborhood grows by $4 s$ and its area grows by $E(j, k, s)=$ $s(2+j+k+2 s)$.

Proof. The neighborhood grows linearly, being equal to $j+k+4 . E(j, k, s)$ is defined as the number of tiles added to a simple shape by $s$ expansions, therefore : $E(j, k, s)=\sum_{i=0}^{s-1}((j+2 i)+(k+2 i)+4)=s(2+j+k+2 s)$.

\subsection{Spines}

Definition 6. A simple shape such that $a \in\{1,2\}$ is called a spine.

Theorem 3. A simple shape $A$ can be described as a spine, expanded some finite number (possibly zero) of times. This description is unique.

We shall next show that there are only 4 kinds of spines. Thus, since we know the area added by each expansion step, we can calculate the areas of all simple shapes.

Proof. Of the theorem. If $a$ of $A$ is even, we say that $A_{s}$ has dimensions $2, b-a+2$, otherwise $1, b-a+1$. Either way, $A_{s}$ is a spine and expanding it $s=\left\lceil\frac{a}{2}\right\rceil-1$ times yields exactly $A$. Then the area of every simple shape is the sum of the area of its spine $A_{s}$ and the area added in the expansions. We note that starting from any other spine will result in the wrong shape - a different initial width (or different number of expansions) results in wrong parity of the final width, and the same spine width but different different length results in a wrong difference between length and width. Therefore this description is unique.

Lemma 4. Let $A_{s}$ be a spine of dimensions $a \leq b$, then its area is given by (See Figure 5]):

1. If $a=1$, the area is $b+1$

2. If $a=2$, then we have the following options: 
(a) If $b$ is odd, then $\left|A_{s}\right|=\frac{3 \cdot(b+1)}{2}$.

(b) $b$ is even, of type 1 , then $\left|A_{s}\right|=\frac{3 \cdot b}{2}+1$.

(c) $b$ is even, of type 2, then $\left|A_{s}\right|=\frac{3 \cdot b}{2}+2$.

Proof. For $a=1$, there are $b$ tiles at distances 0 to $b-1$ from one line, and one more. For $a=2$, there are $\left\lfloor\frac{b+1}{2}\right\rfloor$ triplets of tiles. Note that there are two ways of getting from an odd $b$ to an even one, depending on which boundary is moved, resulting in different area increases.

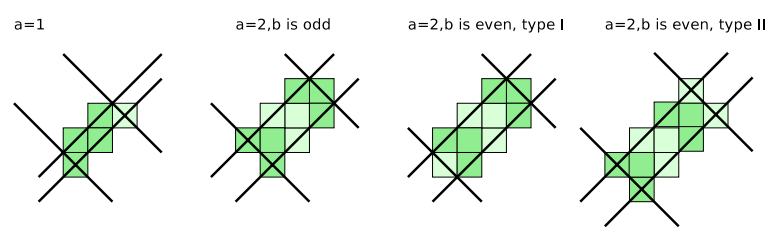

Fig. 5. Spine types and their areas

\subsection{Spines of Optimal Shapes}

Theorem 4. Let $A_{s}$ be a spine with dimension $a, b$ of an optimal shape $A$, then $a+4>b$.

Later we show that this result, while necessary in our construction, is not tight.

Proof. We assume by contradiction that $A$ is an optimal shape with spine $a+4 \leq$ $b$, extended $s$ times. Then we take the same skeleton with $b$ shortened by 4 , and expanding it $s+1$ times we get $B$, such that $|N(A)|=|N(B)|$.

We will now show that $|B|>|A|$, contradicting the optimality of $A$. $|A|$ is the sum of spine size and $E(a, b, s)$.

First we note that $E(a, b-4, s+1)-(E(a, b, s))=a+b$, then if $a=1$, the area of the skeletons is $b-4+1$ and $b+1$, then $b-4+a \geq a+4-4+a>0 \Rightarrow$ $(b-4+1)+(a+b)>b+1$.

If $a=2$, in all the variations, subtracting 4 from $b$ reduces the skeleton area by precisely 2 , but the expansions more than offset that because $a+b \geq 2 a+$ $4>2$.

Corollary 1. The dimensions of spines of optimal shapes are a subset of:

$$
\{(1,1),(1,2),(1,3),(1,4),(2,2),(2,3)(2,4),(2,5)\}
$$

Recalling Lemma 4, we note that spines of dimensions $\{(2,2),(2,4)\}$ mentioned above come in two types. As we saw then type 2 spines have strictly more area than those of type 1 , with the same neighborhood. Therefore only type 2 spines can result in optimal shapes. In this context, each set of spine dimensions results in a certain spine area and neighborhood size. 
Theorem 5. Let $A$ be a non degenerate optimal shape with dimensions $j, k$, so that $|N(A)|=4(m+1)+i$, with $i \in\{0,1,2,3\}$. Then:

$$
|A|=2 m^{2}+(1+i) m+\max \{1, i\}
$$

Proof. Let $a, b$ be the dimensions of $A$ 's spine, then remembering each expansion increases the neighborhood size by 4 , we see that $4(m+1)+i=j+k+4=$ $a+b+4(s+1)$. One conclusion is that $a+b \equiv i \bmod 4$, and another is that $s=\frac{4 m+i-a-b}{4}$. Hence, denoting $\left|A_{s}\right|$ the area of the skeleton of dimensions $a, b$, the total area for such a shape is exactly $|A|=\left|A_{s}\right|+E\left(a, b, \frac{4 m+i-a-b}{4}\right)$.

Below we have a table describing for each $i$ the possible spines for optimal shapes with $|N(A)|=4 m+i$, the shape's area for each spine, and the spines resulting in shapes that are sub-optimal for that neighborhood size.

\begin{tabular}{|c|c|c|c|c|c|c|}
\hline$i$ & Spine & Spine Type & Spine Area & \# of Expansions & Total Area & Sub-opt. \\
\hline \hline 0 & $(1,3)$ & $a=1$ & $3+1$ & $m-1$ & $2 m^{2}+2 m$ & yes \\
\hline 0 & $(2,2)$ & $a=2 b$ is even & $\frac{3 \cdot 2}{2}+2$ & $m-1$ & $2 m^{2}+2 m+1$ & \\
\hline 1 & $(1,4)$ & $a=1$ & $4+1$ & $m-1$ & $2 m^{2}+3 m$ & yes \\
\hline 1 & $(2,3)$ & $a=2 b$ is odd & $\frac{3 \cdot(3+1)}{2}$ & $m-1$ & $2 m^{2}+3 m+1$ & \\
\hline 2 & $(1,1)$ & $a=1$ & $1+1$ & $m$ & $2 m^{2}+4 m+2$ & \\
\hline 2 & $(2,4)$ & $a=2 b$ is even & $\frac{3 \cdot 4}{2}+2$ & $m-1$ & $2 m^{2}+4 m+2$ & \\
\hline 3 & $(1,2)$ & $a=1$ & $2+1$ & $m$ & $2 m^{2}+5 m+3$ & \\
\hline 3 & $(2,5)$ & $a=2 b$ is odd & $\frac{3 \cdot(5+1)}{2}$ & $m-1$ & $2 m^{2}+5 m+2$ & yes \\
\hline
\end{tabular}

Theorem 5 5 provided a necessary condition for an optimal non degenerate simple shape $A$. However, although we have shown the optimal area for every specific neighborhood size, we are not done yet. We must show that no shape exists having larger area and smaller neighborhood. This should hold because $n(k)$ is defined so that it is a non-decreasing sequence.

First we note that for any specific $m,|A|$ is strictly monotonous in $i$. Furthermore, we see that $2(m+1)^{2}+2(m+1)+1=2 m^{2}+4 m+2+2 m+2+1=$ $2 m^{2}+6 m+5>2 m^{2}+5 m+3$, then $|A|$ is strictly monotonous in $N(A)$. Thus, all size values in the above result are indeed areas of optimal shapes. Therefore:

Theorem 6. The non-degenerate optimal shapes are those simple shapes that when decomposed into spine and expansion have a spine of one of the following forms: $(a, b) \in\{(1,1),(1,2),(2,2),(2,3)(2,4)\}$ (these spines appear in Figure [6]).

Corollary 2. Let $A_{s}$ be a spine with dimension $a, b$ of an optimal shape $A$, then $a+3>b$.

Corollary 2 is a tighter version of theorem 4 , and can now be verified by inspection of the list of optimal spines.

Corollary 3. The degenerate simple shapes with areas $0,1,2$ are all optimal.

All these appear in Figure 7. 


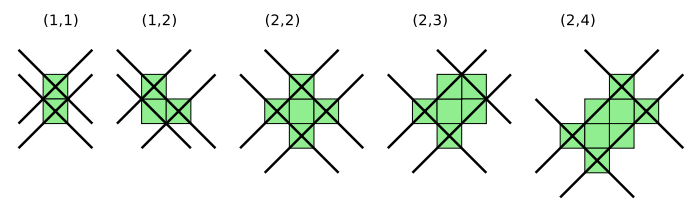

Fig. 6. The optimal spines

Proof. There are no other optimal shapes with neighborhoods sizes 0 or 4 , and the other optimal shapes of neighborhood size 6 also have area equal to 2 .

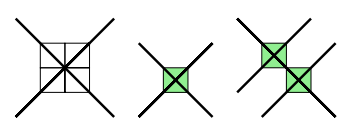

Fig. 7. The optimal degenerate shapes: two with dimensions $(0,0)$ and one with $(0,2)$

We have now identified all the optimal shapes, degenerate and simple, with explicit expressions for their neighborhood sizes and areas. This allows us to state that every shape of area $k$ has a neighborhood at least as large as that of (every) optimal shape with area $\leq k$. From this characterization, Theorem 1 immediately follows.

\section{Alternative Analysis}

This section describes an alternative approach to the grid isoperimetric inequality. Some results similar to those presented in section 2 are rederived, as well as a new result, concerning the 8-connectivity grid metric.

\subsection{Four Connectivity in $\mathbb{Z}^{2}$}

Let $A$ be a finite subset of the $\mathbb{Z}^{2}$ grid, having the neighborhood $N(A)=$ $\left\{p \in \mathbb{Z}^{2} \mid d(p, A)=1\right\}$, where $d$ is the Manhattan metric $d((a, b),(c, d))=|d-b|$ $+|c-a|$. Let us denote $n(A)=|N(A)|$.

For some area $k \in \mathbb{N}$ let $A_{M I N}(k)$ be defined as the shape of area $k$ whose neighborhood is the smallest, namely :

$$
\begin{aligned}
& A_{M I N}(k) \subset \mathbb{Z}^{2} \wedge\left|A_{M I N}(k)\right|=k \wedge \\
& \forall A \subset \mathbb{Z}^{2} \quad(|A|=k) \rightarrow\left(n(A) \geq n\left(A_{M I N}(k)\right)\right)
\end{aligned}
$$

Theorem 7. For every positive $k$, the neighborhood of $A_{M I N}(k)$ is at least as large as this of the largest digital sphere (assuming 4 Connectivity) of size at most $k$, minus two, namely:

$$
\forall k \in \mathbb{N} \quad n\left(A_{M I N}(k)\right) \geq \max \left\{n\left(A_{S P H E R E}\right)|| A_{S P H E R E} \mid \leq k\right\}-2
$$


We provide only the outline of this proof due to space considerations, but the steps described here are followed in the proof for 8 Connectivity. As we proved before, any shape $A$ is covered by a simple shape $R$ that has no more neighbours than $A$. This simple shape $R$ is enclosed by another simple shape denoted $C R$, that has all of its neighbours on tiles such that the parity of the sum of their coordinates is constant (if the plane is colored as a chess board, the neighbours are all of the same color) and such that $C R$ has at most two more neighbours than $C$ and $A$. We call such a shape a Canonical Rectangle. We derive formulae for the area and boundary size of $C R$ based on the sizes of its sides $a, b$, then find a lower bound on the minimal boundary for a canonical rectangle of the same area by differentiating by $b$. This minimum is found when $a=b$, that is when the canonical rectangle is a Manhattan sphere.

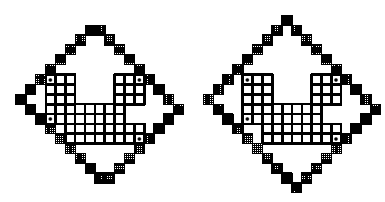

Fig. 8. For a shape $A$, the left chart demonstrates $R$ while the right chart demonstrates the canonical rectangle $C R$

\subsection{Eight Connectivity in $\mathbb{Z}^{2}$}

Let $B$ be a finite subset of the $\mathbb{Z}^{2}$ grid, having the neighborhood $N_{8}(B)=$ $\left\{p \in \mathbb{Z}^{2} \mid d_{8}(p, B)=1\right\}$, where $d_{8}((a, b),(c, d))=\max \{(d-b),(c-a)\}$. Let us denote $n_{8}(B)=\left|N_{8}(B)\right|$.

For some area $k \in \mathbb{N}$ let $B_{M I N}(k)$ be defined as the shape of area $k$ whose neighborhood is the smallest, namely :

$$
\begin{aligned}
& B_{M I N}(k) \subset \mathbb{Z}^{2} \wedge\left|B_{M I N}(k)\right|=k \wedge \\
& \forall B \subset \mathbb{Z}^{2} \quad(|B|=k) \rightarrow\left(n_{8}(B) \geq n_{8}\left(B_{M I N}(k)\right)\right)
\end{aligned}
$$

Theorem 8. For every positive $k$, the size of the neighborhood of $B_{M I N}(k)$ is at least as large as this of the largest digital sphere (assuming 8 Connectivity) of size at most $k$, namely :

$$
\forall k \in \mathbb{N} \quad n_{8}\left(B_{M I N}(k)\right) \geq \max \left\{n_{8}\left(B_{S P H E R E}\right)|| B_{S P H E R E} \mid \leq k\right\}
$$

Note that a digital sphere of radius 3, for example, is a 5 by 5 square.

Proof. Let us denote the bounding rectangle of $B$ by bounding-rectangle $(B)$. For each of the four sides of bounding-rectangle $(B)$ (i.e. top, right, down, left) let us denote the last tiles of $B$ that are 4 neighbors of the four sides (assuming clockwise movement) by 1, 2, 3 and 4 respectively. See an example in Figure 9.

Let us project all the tiles of bounding-rectangle $(B)$ between points 1 and 2 in $45^{\circ}$ down-left, the points between 2 and 3 in $45^{\circ}$ up-left, the points between 

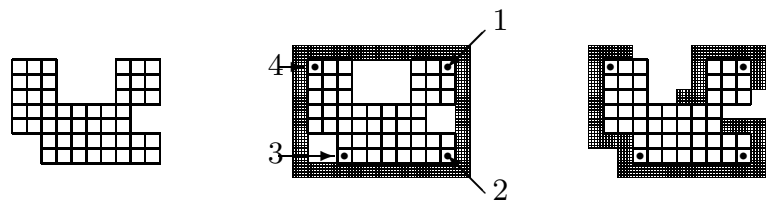

Fig. 9. An example of the bounding-rectangle and its projection

3 and 4 in $45^{\circ}$ up-right and the points between 4 and 1 in $45^{\circ}$ down-right. An example appears in Figure 9.

Clearly, after such projection each tile of bounding-rectangle $(B)$ a 4 neighbor of at least a single tile of $B$. In addition, it is impossible that two tiles of boundingrectangle $(B)$ will merge in the same spot. Thus, $n_{8}(B)$ is at least the number of tiles in bounding-rectangle $(B)$, namely :

$$
\forall B \quad n_{8}(B) \geq \mid \text { bounding-rectangle }(B) \mid
$$

Let $R(k)$ be the smallest rectangle which contains at least $k$ tiles. Let $a$ and $b$ denote the sides of $R(k)$ and let $c$ denote the number of tiles $R(k)$ comprises. Then :

$$
c=2(a+b)-4
$$

Let $f(a, b)$ denote the area of a rectangle of sides $a$ and $b$ :

$$
f(a, b)=(a-2)(b-2)
$$

We would like to find a solution for the following optimization problem :

$$
\min \quad c \quad \text { s.t } \quad f(a, b) \geq k \quad \wedge \quad c=2(a+b)-4
$$

After some arithmetics equation 3 can be written as :

$$
a=\frac{f(a, b)}{b-2}+2
$$

Combining this with 2 we get $c=\frac{2 f(a, b)}{b-2}+2 b$. Since we require that $f(a, b) \geq k$ we can write the following :

$$
c \geq \rho \triangleq \frac{2 k}{b-2}+2 b
$$

Note that while the minimizing $b$ may not be an integer, it still gives a bound valid over the integer $b$. In order to minimize $\rho$ we require that $\frac{\partial \rho}{\partial b}=2-\frac{2 k}{(b-2)^{2}}=0$ and after some arithmetics we get that:

$$
b=\sqrt{k}+2
$$

By examining the behavior of $\frac{\partial^{2} \rho}{\partial b^{2}}$ we can see that for $b=\sqrt{k}+2$ since $k \geq 1$ then $\frac{\partial^{2} \rho}{\partial b^{2}}>0$, meaning that $\rho$ is indeed minimized at this point. By assigning the 
value of $b_{\min }$ to equations 4 and 5 we can see that for $b_{\min }, a=b$ (meaning that $R(k)$ is a square - the equivalent of a digital sphere, assuming 8 Connectivity) and that :

$$
c \geq 4(\sqrt{k}+1)
$$

It is easy to see that for some sphere $B$ such that $|B|=k, n_{8}(B)=4(\sqrt{k}+1)$ and therefore it is the shape that minimizes the neighborhood for shapes of given area $k$. The rest of the Theorem is implied.

\section{References}

1. Wang, D.L., Wang, P.: Discrete Isoperimetric Problems. SIAM J. Appl. Math., 32(4) (1977) $860-870$

2. Bezrukov, S.L.: Isoperimetric Problems in Discrete Spaces. Extremal Problems for Finite Sets, Janos Bolyai Soc. Math. Stud. 3, Budapest, Hungary (1994) 59-91

3. Chung, F.: Discrete Isoperimetric Inequalities. Surveys in Differential Geometry IX, International Press (2004) 53-82

4. Bollob'as, B., Radcliffe, A.J.: Isoperimetric Inequalities for Faces of the Cube and the Grid. Europ. J. Combinatorics, 11 (1990) 323-333

5. Tiersma, H.J.: A Note on Hamming Spheres. Discr. Math., 54 (1985) 225-228

6. Altshuler, Y., Bruckstein, A.M., Wagner, I.A.: Swarm Robotics for a Dynamic Cleaning Problem. IEEE Swarm Intelligence Symposium (2005) 209-216 